Generalized adiabatic product expansion: A nonperturbative method of solving the time-dependent Schrödinger equation

Ali Mostafazadeh

Citation: Journal of Mathematical Physics 40, 3311 (1999); doi: 10.1063/1.532889

View online: http://dx.doi.org/10.1063/1.532889

View Table of Contents: http://aip.scitation.org/toc/jmp/40/7

Published by the American Institute of Physics

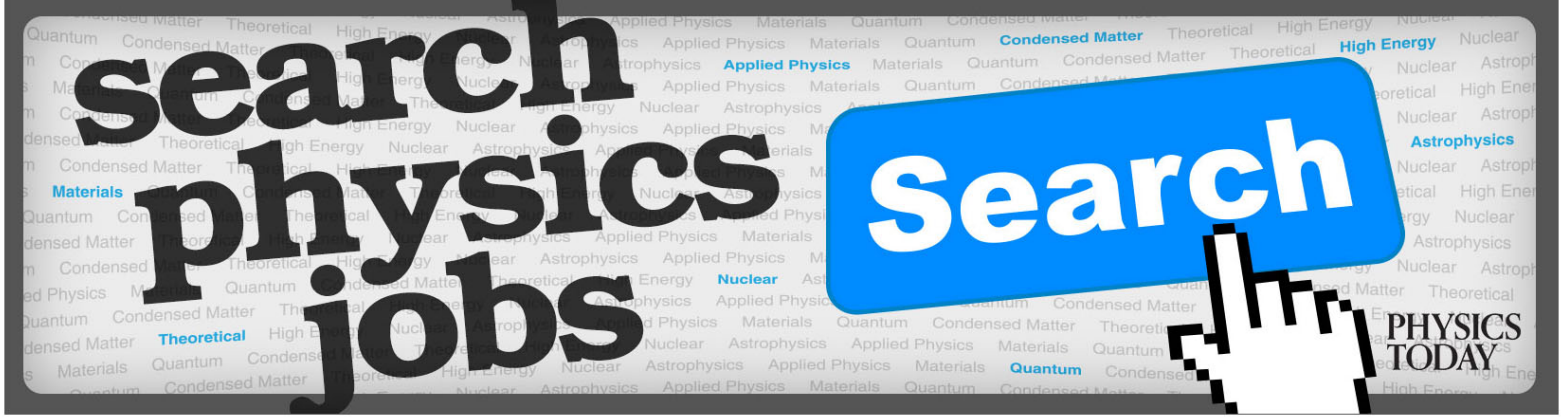




\title{
Generalized adiabatic product expansion: A nonperturbative method of solving the time-dependent Schrödinger equation
}

\author{
Ali Mostafazadeh ${ }^{\text {a) }}$ \\ Department of Mathematics, Koç University, Istinye 80860 Istanbul, Turkey
}

(Received 6 January 1999; accepted for publication 17 March 1999)

We outline a method based on successive canonical transformations which yields a product expansion for the evolution operator of a general (possibly non-Hermitian) Hamiltonian. For a class of such Hamiltonians this expansion involves a finite number of terms, and our method gives the exact solution of the corresponding time-dependent Schrödinger equation. We apply this method to study the dynamics of a general nondegenerate two-level quantum system, a time-dependent classical harmonic oscillator, and a degenerate system consisting of a spin 1 particle interacting with a time-dependent electric field $\overrightarrow{\mathcal{E}}(t)$ through the Stark Hamiltonian $H$ $=\lambda(\vec{J} \cdot \overrightarrow{\mathcal{E}})^{2}$. (C) 1999 American Institute of Physics. [S0022-2488(99)02207-0]

\section{INTRODUCTION}

Recently, a method based on successive canonical transformations has been used to obtain exact solution of the Schrödinger equation

$$
i \frac{d}{d t}|\psi(t)\rangle=H(t)|\psi(t)\rangle
$$

for a class of dipole Hamiltonians ${ }^{1-3}$ and time-dependent harmonic oscillators. ${ }^{4}$ For these systems the Hamiltonian is a nondegenerate Hermitian operator. The purpose of the present article is to extend the application of this method to the cases where the Hamiltonian is non-Hermitian and involves degenerate eigenvalues.

Non-Hermitian Hamiltonians have been used to model a variety of physical systems involving decaying states. ${ }^{5}$ The solution of the Schrödinger equation for a time-dependent two-level nonHermitian Hamiltonian has been considered in Refs. 6 and 7. Another motivation for the study of the Schrödinger equation for a time-dependent non-Hermitian Hamiltonian is the fact that the solution of every linear ordinary differential equation (ODE) may be reduced to the solution of a system of first-order linear ODEs which can be written in the form of the time-dependent Schrödinger equation (1) or alternatively

$$
\begin{gathered}
|\psi(t)\rangle=U(t)|\psi(0)\rangle, \\
i \frac{d}{d t} U(t)=H(t) U(t), \\
U(t)=1,
\end{gathered}
$$

where $U(t)$ is the evolution operator. For a general linear ODE the corresponding Hamiltonian $H(t)$ may be a non-Hermitian matrix with degenerate eigenvalues.

${ }^{a)}$ Electronic mail: amostafazadeh@ku.edu.tr 
The method of adiabatic product expansion developed in Refs. 1 and 2 does not directly apply to quantum systems with non-Hermitian Hamiltonians. In this article we shall present a generalization of this method which applies to arbitrary (possibly) non-Hermitian Hamiltonians with degenerate as well as nondegenerate eigenvalues.

The organization of the article is as follows. In Sec. II we review the basic results concerning the adiabatic approximation for degenerate and non-Hermitian Hamiltonians. In Sec. III we discuss the generalization of the method of adiabatic product expansion to these Hamiltonians. In Sec. IV we use the results of Sec. III to study the solution of the Schrödinger equation for a general nondegenerate non-Hermitian two-level Hamiltonian. In Sec. V, we apply the general results obtained in Sec. IV to treat the classical equation of motion for a harmonic oscillator with a time-dependent frequency. In Sec. VI, we discuss the application of the adiabatic product expansion to study the quadrupole interaction of a spin 1 particle with a time-dependent electric field $\overrightarrow{\mathcal{E}}=\left(\mathcal{E}_{1}(t), \mathcal{E}_{2}(t), 0\right)$. We show that the corresponding Hamiltonian which has a degenerate and a nondegenerate eigenvalue is canonically equivalent to a Hamiltonian which has only nondegenerate eigenvalues. Furthermore, we show that if the direction of the electric field depends in a particular way on its magnitude, then our method yields the exact solution of the Schrödinger equation. Finally we present our conclusions in Sec. VII.

\section{ADIABATIC APPROXIMATION FOR NON-HERMITIAN HAMILTONIANS}

Let $H=H[R]$ be a parametric Hamiltonian which depends on a set of real parameters $R$ $=\left(R^{1}, R^{2}, \ldots, R^{d}\right)$ labeling the points of a smooth manifold $M$. Let $E_{n}[R]$ denote the eigenvalues of $H[R]$ and $\mathcal{H}_{n}[R]$ be the degeneracy subspace associated with $E_{n}[R]$. Let $\mathcal{N}$ denote the degree of degeneracy of $E_{n}[R]$, i.e., the complex dimension of $\mathcal{H}_{n}[R]$. We shall assume that the spectrum of $H[R]$ is discrete and $\mathcal{N}$ does not depend on $R$.

Now let $\left|\psi_{n}, a ; R\right\rangle$ and $\left|\phi_{n}, a ; R\right\rangle$ form a complete biorthonormal basis of the Hilbert space. ${ }^{8,9}$ This means that $\left|\psi_{n}, a ; R\right\rangle$ with $a \in\{1,2, \ldots, \mathcal{N}\}$ form a basis of $\mathcal{H}_{n}[R]$, in particular

$$
H[R]\left|\psi_{n}, a ; R\right\rangle=E_{n}[R]\left|\psi_{n}, a ; R\right\rangle,
$$

and $\left|\phi_{n}, a ; R\right\rangle$ satisfy

$$
\begin{gathered}
H[R]^{\dagger}\left|\phi_{n}, a ; R\right\rangle=E_{n}^{*}[R]\left|\phi_{n}, a ; R\right\rangle, \\
\left\langle\phi_{m}, b ; R \mid \psi_{n}, a ; R\right\rangle=\delta_{m n} \delta_{a b}, \\
\sum_{n} \sum_{a=1}^{\mathcal{N}}\left|\psi_{n}, a ; R\right\rangle\left\langle\phi_{n}, a ; R\right|=1 .
\end{gathered}
$$

Next suppose that the parameters $R^{i}$ depend on time $t$, then $R(t)$ defines a curve $\mathcal{C}$ in the parameter space $M$, and the Hamiltonian, its eigenvalues, and eigenvectors become time dependent. In this case we use the notation $H(t):=H[R(t)], E_{n}(t):=E_{n}[R(t)], \quad\left|\psi_{n}, a ; t\right\rangle$ $:=\left|\psi_{n}, a ; R(t)\right\rangle$, and $\left|\phi_{n}, a ; t\right\rangle:=\left|\phi_{n}, a ; R(t)\right\rangle$. We shall assume that $E_{n}(t),\left|\psi_{n}, a ; t\right\rangle$ and $\left|\phi_{n}, a ; t\right\rangle$ are smooth functions of $t$ and that during the evolution of the system the eigenvalues of the Hamiltonian do not cross, i.e., if $E_{m}(0)<E_{n}(0)$, then for all $t \in[0, \tau], E_{m}(t)<E_{n}(t)$, where $\tau$ denotes the duration of the evolution of the system.

Differentiating both sides of Eq. (5) with respect to $t$, taking the inner product of both sides of the resulting equation with $\left|\phi_{m}, b ; t\right\rangle$, for arbitrary $m$ and $b$, and using Eqs. (5)-(7), we have

$$
\left[E_{m}(t)-E_{n}(t)\right]\left\langle\phi_{m}, b ; t\left|\frac{d}{d t}\right| \psi_{n}, a ; t\right\rangle+\left\langle\phi_{m}, b ; t|\dot{H}(t)| \psi_{n}, a ; t\right\rangle-\delta_{m n} \delta_{a b} \dot{E}(t)=0
$$

Here a dot denotes differentiation with respect to $t$. For $m \neq n$, Eq. (9) reads 


$$
\left\langle\phi_{m}, b ; t\left|\frac{d}{d t}\right| \psi_{n}, a ; t\right\rangle=\frac{\left\langle\phi_{m}, b ; t|\dot{H}(t)| \psi_{n}, a ; t\right\rangle}{E_{n}(t)-E_{m}(t)} \text { for } m \neq n \text {. }
$$

Now let us express the solution of the Schrödinger equation (1) in the basis $\left\{\left|\psi_{n}, a ; t\right\rangle\right\}$. Then

$$
|\psi(t)\rangle=\sum_{n} \sum_{a=1}^{\mathcal{N}} C_{a}^{n}(t)\left|\psi_{n}, a ; t\right\rangle,
$$

where $C_{a}^{n}(t)$ are complex coefficients. Substituting Eq. (11) in the Schrödinger equation (1), taking the inner product of both sides of the resulting equation with $\left|\phi_{m}, b ; t\right\rangle$, and making use of Eqs. (5), (6), (7), and (10), we find

$$
i \dot{C}_{b}^{m}-E_{m} C_{b}^{m}+\sum_{a=1}^{\mathcal{N}} i\left\langle\phi_{m}, b ; t \mid \frac{d}{d t} \psi_{m}, a ; t\right\rangle C_{a}^{m}=-i \sum_{n \neq m} \sum_{a=1}^{\mathcal{N}} \frac{\left\langle\phi_{m}, b ; t|\dot{H}(t)| \psi_{n}, a ; t\right\rangle}{E_{n}(t)-E_{m}(t)} .
$$

The special case of this equation with $\mathcal{N}=1$, i.e., the nondegenerate case, has been originally derived by Garrison and Wright ${ }^{9}$ in their investigation of the adiabatic geometric phase ${ }^{10}$ for non-Hermitian Hamiltonians., ${ }^{91-14}$

If the right-hand side of Eq. (12) is negligible, then one says that the system undergoes an adiabatic evolution. ${ }^{15,16,2,9,17}$ In this case, the equations for $C_{a}^{n}$ decouple and their solution is given by

$$
C_{a}^{n}(t)=\sum_{b=1}^{\mathcal{N}} K_{a b}^{n}(t) C_{b}^{n}(0)
$$

where $K_{a b}^{n}(t)$ are entries of the invertible matrix

$$
K^{n}(t):=e^{-i \int_{0}^{t} E_{n}(s) d s} \mathcal{P} \exp \left[i \int_{R(0)}^{R(t)} \mathcal{A}^{n}[R]\right],
$$

$\mathcal{P}$ denotes the path-ordering operator, $\mathcal{A}^{n}$ is the matrix of one-forms with entries

$$
\mathcal{A}_{a b}^{n}[R]:=i\left\langle\phi_{n}, a ; R|d| \psi_{n}, b ; R\right\rangle,
$$

$d$ stands for the exterior derivative with respect to $R^{i}$, and the line integral in Eq. (14) is evaluated along the curve $\mathcal{C}$ defined by $R(t)$. If $\mathcal{C}$ is a closed curve in $M$, the Hamiltonian has a periodic time dependence and the path-ordered exponential in Eq. (14), which takes the form

$$
\mathcal{P} \exp \left[i \oint_{\mathcal{C}} \mathcal{A}^{n}[R]\right],
$$

is the non-Hermitian analog of the non-Abelian adiabatic geometric phase. ${ }^{18}$

Note that if the initial vector $|\psi(0)\rangle$ is an eigenvector of the initial Hamiltonian $H(0)$, then the adiabaticity of the evolution implies that $|\psi(t)\rangle$ is an eigenvector of $H(t)$ for all $t \in[0, \tau]$. In terms of the time-evolution operator $U(t)$ of Eq. (3) this is expressed by

$$
U(t) \approx U^{(0)}(t),
$$

where

$$
U^{(0)}(t):=\sum_{n} \sum_{a, b=1}^{\mathcal{N}} K_{a b}^{n}(t)\left|\psi_{n}, a ; t\right\rangle\left\langle\phi_{n}, b ; 0\right| .
$$


One can easily show that $U^{(0)}(t)$ is invertible, and its inverse is given by

$$
U^{(0)^{-1}}(t)=\sum_{n} \sum_{a, b=1}^{\mathcal{N}} K_{a b}^{n^{-1}}(t)\left|\psi_{n}, a ; 0\right\rangle\left\langle\phi_{n}, b ; t\right|
$$

where $K^{n^{-1}}(t)$ is the inverse of $K^{n}(t)$.

\section{ADIABATIC CANONICAL TRANSFORMATIONS AND THE GENERALIZED ADIABATIC PRODUCT EXPANSION}

Let $g(t)$ be an invertible linear operator acting on the Hilbert space. Then the transformations:

$$
\begin{gathered}
|\psi(t)\rangle \rightarrow\left|\psi^{\prime}(t)\right\rangle:=g(t)|\psi(t)\rangle, \\
H(t) \rightarrow H^{\prime}(t):=g(t) H(t) g(t)^{-1}-i g(t) \frac{d}{d t} g(t)^{-1}, \\
U(t) \rightarrow U^{\prime}(t):=g(t) U(t) g(0)^{-1},
\end{gathered}
$$

leave the form of the Schrödinger equation invariant. We shall call such a transformation a canonical transformation.

Now let us investigate the consequences of the canonical transformation defined by $g(t)$ $=U^{(0)}(t)^{-1}$. We shall call this transformation the adiabatic canonical transformation. Denoting the transformed Hamiltonian $H^{\prime}$ by $H^{(1)}$, we have

$$
H^{(1)}(t)=\sum_{n, m \neq n} \sum_{a=1}^{\mathcal{N}} \sum_{b=1}^{\mathcal{M}} H_{a b}^{(1)^{n m}}(t)\left|\psi_{n}, a ; 0\right\rangle\left\langle\phi_{m}, b ; 0\right|,
$$

where

$$
H_{a b}^{(1) n m}(t):=-K_{a c}^{(n)}(t)^{-1} A_{c d}^{n m}(t) K_{d b}^{m}(t), \quad A_{c d}^{n m}(t):=i\left\langle\phi_{n} c ; t\left|\frac{d}{d t}\right| \psi_{m}, d ; t\right\rangle .
$$

Because $g(0)=U^{(0)}(0)^{-1}=1$, the transformed evolution operator is given by

$$
U^{\prime}(t)=U^{(0)}(t)^{-1} U(t)
$$

Clearly if the adiabatic approximation is valid, $H^{(1)}(t) \approx 0$ and $U^{\prime}(t) \approx 1$.

Let us suppose that $H^{(1)}(t)$ has a discrete spectrum and denote by $E_{n_{1}}^{(1)}(t)$ and $\mathcal{N}_{1}$ the eigenvalues of $H^{(1)}(t)$ and their degree of degeneracy. Furthermore, let $\left\{\left|\psi_{n_{1}}^{(1)}, a_{1} ; t\right\rangle,\left|\phi_{n}^{(1)}, a_{1} ; t\right\rangle\right\}$ be a biorthonormal eigenbasis of the Hilbert space, i.e.,

$$
\begin{gathered}
H^{(1)}(t)\left|\psi_{n_{1}}^{(1)}, a_{1} ; t\right\rangle=E_{n_{1}}^{(1)}(t)\left|\psi_{n_{1}}^{(1)}, a_{1} ; t\right\rangle \\
H^{(1)}(t)^{\dagger}\left|\phi_{n_{1}}^{(1)}, a_{1} ; t\right\rangle=E_{n_{1}}^{(1) *}(t)\left|\phi_{n_{1}}^{(1)}, a_{1} ; t\right\rangle \\
\left\langle\phi_{m_{1}}^{(1)}, b_{1} ; t \mid \psi_{n_{1}}^{(1)}, a_{1} ; t\right\rangle=\delta_{m_{1} n_{1}} \delta_{a_{1} b_{1}}, \quad \sum_{n_{1}} \sum_{a_{1}=1}^{\mathcal{N}_{1}}\left|\psi_{n_{1}}^{(1)}, a_{1} ; t\right\rangle\left\langle\phi_{n_{1}}^{(1)}, a_{1} ; t\right|=1 .
\end{gathered}
$$

Then $H^{(1)}(t)$ shares the properties of the original Hamiltonian $H(t)$, and we can repeat the above analysis using $H^{(1)}(t)$ in place of $H(t)$. In this way the adiabatic approximation yields the approximate evolution operator 


$$
U^{(1)}(t)=\sum_{n_{1}} \sum_{a_{1}, b_{1}=1}^{\mathcal{N}_{1}} K_{a_{1} b_{1}}^{(1) n_{1}}(t)\left|\psi_{n_{1}}^{(1)}, a_{1} ; t\right\rangle\left\langle\phi_{n_{1}}^{(1)}, b_{1} ; 0\right|
$$

for $H^{(1)}(t)$, where $K_{a_{1} b_{1}}^{(1) n_{1}}(t)$ are the entries of the matrix $K^{(1) n_{1}}$ obtained by replacing $E_{n}(t)$, $\left|\psi_{n}, a ; t\right\rangle$ and $\left|\phi_{n}, a ; t\right\rangle$ in Eqs. (14) and (15) by $E_{n_{1}}^{(1)}(t),\left|\psi_{n_{1}}^{(1)}, a_{1} ; t\right\rangle$, and $\left|\phi_{n_{1}}^{(1)}, a_{1} ; t\right\rangle$, respectively.

Next we perform the adiabatic canonical transformation defined by $g(t)=U^{(1)}(t)^{-1}$. This leads to a transformed Hamiltonian $H^{(2)}(t)$ which is related to $H^{(1)}(t)$ according to Eqs. (23) and (24) with $K^{n},\left|\psi_{n}, a ; t\right\rangle$, and $\left|\phi_{n}, b ; t\right\rangle$ replaced by $K^{(1) n_{1}},\left|\psi_{n_{1}}^{(1)}, a_{1} ; t\right\rangle$, and $\left|\phi_{n_{1}}^{(1)}, a_{1} ; t\right\rangle$. The transformed evolution operator is given by

$$
U^{(1)}(t)^{-1} U^{(0)}(t)^{-1} U(t)
$$

Repeating this procedure we obtain, after $N$ successive adiabatic canonical transformations, a transformed Hamiltonian $H^{(N)}(t)$ and a transformed evolution operator which is given by

$$
U^{(N-1)}(t)^{-1} U^{(N-2)}(t)^{-1} \cdots U^{(0)}(t)^{-1} U(t) .
$$

Here $U^{(\ell)}(t)$, with $\ell \in\{1,2, \ldots, N-1\}$, denotes the approximate evolution operator obtained by performing adiabatic approximation on the Hamiltonian $H^{(\ell)}(t)$.

If for some $N$ the adiabatic approximation yields the exact solution of the Schrödinger equation for the Hamiltonian $H^{(N)}(t)$, then by construction $H^{(N+1)}(t)=0$ and $U^{(N+1)}(t)=1$. In this case, the original evolution operator is given by

$$
U(t)=U^{(0)}(t) U^{(1)}(t) \cdots U^{(N)}(t) .
$$

If the adiabatic approximation fails for all $H^{(N)}(t)$, then there are two possibilities

(i) One obtains an infinite product expansion for the evolution operator

$$
U(t)=\prod_{\ell=0}^{\infty} U^{(\ell)}(t):=U^{(0)}(t) U^{(1)}(t) \cdots U^{(\ell)}(t) \cdots .
$$

In this case, one may view Eq. (27) as a generalization of the adiabatic approximation.

(ii) One obtains $H^{(i)}(t)=H^{(j)}(t)$ for some $i$ and $j$ with $i \neq j$. In this case a direct application of the method of adiabatic product expansion does not produce a solution. However, as we shall see in Sec. IV, sometimes it is possible to modify this method by combining the adiabatic canonical transformation with other canonical transformations, so that one obtains a finite or an infinite product expansion with distinct terms.

\section{APPLICATION TO TWO-LEVEL HAMILTONIANS}

Two-level nondegenerate Hamiltonians provide the simplest nontrivial quantum systems. This has been one of the main reasons for the study of these Hamiltonians since the early days of quantum mechanics. In this section we shall consider the most general nondegenerate two-level Hamiltonian which may or may not be Hermitian.

In an arbitrary basis of the Hilbert space $\left(\mathrm{C}_{1}^{2}\right)$, the Hamiltonian is given by a two-by-two complex matrix $\bar{H}$. One can perform a quantum canonical transformation (21) defined by $g(t)$ $=\exp \left\{i \int_{0}^{t}[\operatorname{tr} \bar{H}(s)] d s / 2\right\}$ to map the Hamiltonian $\bar{H}$ to a traceless Hamiltonian of the form

$$
H:=\left(\begin{array}{cc}
a & b \\
c & -a
\end{array}\right)
$$


where $\operatorname{tr} \bar{H}$ denotes the trace of $\bar{H}$, and $a=a(t), b=b(t), c=c(t)$ are complex-valued smooth functions of $t$.

We can easily solve the eigenvalue problem for the Hamiltonian (29). The eigenvalues are given by

$$
E_{1}(t):=-E(t), \quad E_{2}(t):=E(t),
$$

where

$$
E:=\sqrt{a^{2}+b c} .
$$

We shall demand that during the time interval $[0, \tau]$ of interest $E \neq 0$, so that the eigenvalues are nondegenerate. In particular, no level crossings occur. Then a possible choice for a biorthonormal eigenbasis is

$$
\begin{gathered}
\left|\psi_{1} ; R\right\rangle=\left(\begin{array}{c}
-b \\
a+E
\end{array}\right), \quad\left|\psi_{2} ; R\right\rangle=\left(\begin{array}{c}
a+E \\
c
\end{array}\right), \\
\left|\phi_{1} ; R\right\rangle=\frac{1}{N^{*}}\left(\begin{array}{c}
-c^{*} \\
a^{*}+E^{*}
\end{array}\right), \quad\left|\phi_{2} ; R\right\rangle=\frac{1}{N^{*}}\left(\begin{array}{c}
a^{*}+E^{*} \\
b *
\end{array}\right),
\end{gathered}
$$

where $R=(a, b, c)$ and $N:=2 E(a+E)$.

Next we compute $U^{(0)}$ and $H^{(1)}$. Using Eqs. (18), (14), (15), and (23), we find

$$
\begin{gathered}
U^{(0)}(t)=K^{1}(t)\left|\psi_{1} ; t\right\rangle\left\langle\phi_{1} ; 0\left|+K^{2}(t)\right| \psi_{2} ; t\right\rangle\left\langle\phi_{2} ; 0\right|, \\
H^{(1)}(t)=\xi(t)\left|\psi_{1} ; 0\right\rangle\left\langle\phi_{2} ; 0|+\zeta(t)| \psi_{2} ; 0\right\rangle\left\langle\phi_{1} ; 0\right|,
\end{gathered}
$$

where

$$
\begin{gathered}
K^{1}(t):=K_{11}^{1}(t)=\exp \left(\frac{i \eta(t)}{2}-\int_{R(0)}^{R(t)}\left[(2 E)^{-1}\left(d a+d E+\frac{c d b}{a+E}\right)\right]\right), \\
K^{2}(t):=K_{11}^{2}(t)=\exp \left(\frac{-i \eta(t)}{2}-\int_{R(0)}^{R(t)}\left[(2 E)^{-1}\left(d a+d E+\frac{b d c}{a+E}\right)\right]\right), \\
\eta(t):=2 \int_{0}^{t} E(s) d s \\
\xi(t):=H_{11}^{12}(t)=\left(-\frac{i e^{-2 i \alpha(t)}}{2}\right)\left[1+\frac{a(t)}{E(t)}\right] \frac{d}{d t}\left[\frac{c(t)}{a(t)+E(t)}\right] \\
\zeta(t):=H_{11}^{21}(t)=\left(\frac{i e^{2 i \alpha(t)}}{2}\right)\left[1+\frac{a(t)}{E(t)}\right] \frac{d}{d t}\left[\frac{b(t)}{a(t)+E(t)}\right] \\
\alpha(t):=\frac{\eta(t)}{2}+\frac{i}{4} \int_{R(0)}^{R(t)} \frac{c d b-b d c}{E(E+a)}
\end{gathered}
$$

The transformed Hamiltonian has the following matrix expression:

$$
H^{(1)}(t)=\left(\begin{array}{cc}
a^{(1)}(t) & b^{(1)}(t) \\
c^{(1)}(t) & -a^{(1)}(t)
\end{array}\right),
$$


where

$$
\begin{gathered}
a^{(1)}(t):=-\frac{b_{0} \xi(t)+c_{0} \zeta(t)}{2 E_{0}}, \\
b^{(1)}(t):=-\frac{b_{0}^{2} \xi(t)-\left(a_{0}+E_{0}\right)^{2} \zeta(t)}{2 E_{0}\left(E_{0}+a_{0}\right)}, \\
c^{(1)}(t):=-\frac{-\left(a_{0}+E_{0}\right)^{2} \xi(t)+c_{0}^{2} \zeta(t)}{2 E_{0}\left(E_{0}+a_{0}\right)},
\end{gathered}
$$

$a_{0}:=a(0), b_{0}:=b(0), c_{0}:=c(0), E_{0}:=E(0)$, and we have used Eqs. (31), (32), and (34).

Note that the transformed Hamiltonian $H^{(1)}(t)$ is traceless, and one can obtain $H^{(2)}(t)$ by substituting $a^{(1)}$ for $a, b^{(1)}$ for $b, c^{(1)}$ for $c$, and $E^{(1)}:=\sqrt{\left(a^{(1)}\right)^{2}+b^{(1)} c^{(1)}}$ for $E$ in Eqs. (34), and (36) - (38). Clearly this can be repeated indefinitely, and one can compute $H^{(\ell)}$ for arbitrary $\ell$.

The adiabatic approximation corresponds to the cases where the matrix elements of $H^{(1)}(t)$ can be neglected. As seen from Eqs. (39) to (42) this happens whenever both $\xi$ and $\zeta$ are negligible. One can also check that if only one of these quantities is negligible, then $H^{(1)}(t)$ is equal to the other times a constant matrix. This means that $H^{(1)}(t)$ has essentially stationary eigenvectors and the adiabatic approximation would yield the solution of the Schrödinger equation for $H^{(1)}$. In fact, it is not difficult to check that for the cases that either $\xi$ or $\zeta$ is negligible, $H^{(2)}(t) \approx 0$. In particular, setting $\xi=0$ or $\zeta=0$ implies $H^{(2)}(t)=0$ and the evolution operator is given by

$$
U(t)=U^{(0)}(t) U^{(1)}(t) .
$$

Therefore, the conditions $\xi=0$ and $\zeta=0$ each define a class of exactly solvable two-level systems. In view of Eqs. (36) and (37), these are as follows.

Class 1: The two-level systems for which $c /(a+E)=\mu=$ constant, or alternatively $c$ $=\mu\left(\mu b+\sqrt{4 a^{2}+\mu^{2} b^{2}}\right) / 2$.

Class 2: The two-level systems for which $b /(a+E)=\nu=$ constant, or alternatively $c=b / \nu^{2}$ $-a^{2} / b$.

In general $\xi$ and $\zeta$ do not vanish and the adiabatic product expansion does not terminate. There is also a special class of two-level systems for which the product expansion has a periodic structure in the sense of case (ii) of Sec. III. This is

Class 3: The two-level systems for which $a=0$.

Setting $a=0$ in Eqs. (38), (35), (36), and (37) and defining $f(t):=i \sqrt{c(t) / b(t)}$, we have

$$
\begin{gathered}
\alpha(t)=\frac{\eta(t)}{2}+\frac{i}{4} \ln \left(\frac{c_{0} b(t)}{b_{0} c(t)}\right), \quad \eta(t)=2 \int_{0}^{t} \sqrt{b(s) c(s)} d s, \\
\xi(t)=-\frac{f_{0} \dot{f}(t) e^{-i \eta(t)}}{2 f(t)}, \quad \zeta(t)=\frac{\dot{f}(t) e^{i \eta(t)}}{2 f_{0} f(t)},
\end{gathered}
$$

where $f_{0}:=f(0)$. Substituting these equations in Eq. (39), we obtain

$$
H^{(1)}(t)=E^{(1)}(t)\left(\begin{array}{cc}
\cos \eta(t) & f_{0}^{-1} \sin \eta(t) \\
f_{0} \sin \eta(t) & -\cos \eta(t)
\end{array}\right),
$$

where

$$
E^{(1)}(t)=\frac{i \dot{f}(t)}{2 f(t)}
$$


This Hamiltonian has two interesting properties.

(1) If $b_{0}=c_{0}$, then $f_{0}=i$ and

$$
H^{(1)}(t)=E^{(1)}(t)\left[\sin \eta(t) \sigma_{2}+\cos \eta(t) \sigma_{3}\right]=E^{(1)}(t) e^{i \eta(t) \sigma_{1} / 2} \sigma_{3} e^{-i \eta(t) \sigma_{1} / 2},
$$

where $\sigma_{i}$ are Pauli matrices, and we have used the identity

$$
e^{-i \varphi \sigma_{i}} \sigma_{j} e^{i \varphi \sigma_{i}}=\cos (2 \varphi) \sigma_{j}+\sin (2 \varphi) \sum_{k=1}^{3} \epsilon_{i j k} \sigma_{k} \text { for } i \neq k
$$

In Eq. (46), $\varphi$ is an arbitrary complex variable and $\epsilon_{i j k}$ is the totally antisymmetric Levi-Civita symbol with $\epsilon_{123}=1$. For the time periods during which $\sqrt{b(t) c(t)}$ is real, $\eta(t)$, is real, and the Hamiltonian (45) is anti-Hermitian. In particular, its eigenvectors are orthogonal. Up to a factor of $i$ this Hamiltonian describes the interaction of a spin 1/2 magnetic dipole with a changing magnetic field. This system has an SU(2) dynamical group. ${ }^{19,20,1,3}$ For the time periods during which $\sqrt{b(t) c(t)}$ is imaginary, $\eta(t)$ is imaginary, and up to a factor of $i$ the Hamiltonian (45) describes a quantum system with a SU(1,1) dynamical group. A Hermitian analog of such a system is the time-dependent generalized harmonic oscillator. ${ }^{21,20,22}$

(2) Performing the adiabatic canonical transformation on (45), we arrive at the unexpected result

$$
H^{(2)}(t)=H(t)
$$

Therefore, direct application of the method of adiabatic product expansion does not lead to a solution.

Next we shall describe a modification of the method of adiabatic product expansion which yields an infinite product expansion for the evolution operator of the Class 3 systems which involve distinct terms.

Consider the transformed Hamiltonian (44). We can express this Hamiltonian using Eq. (39) with

$$
a^{(1)}(t)=E^{(1)}(t) \cos \eta(t), \quad b^{(1)}(t)=f_{0}^{-1} E^{(1)}(t) \sin \eta(t), \quad c^{(1)}(t)=f_{0} E^{(1)}(t) \sin \eta(t) .
$$

Although this Hamiltonian does not belong to Class 3, it can be canonically transformed to a Hamiltonian which belongs to Class 3, i.e., its diagonal matrix elements vanish. This transformation is defined by $g(t)=\exp \left\{i \int_{0}^{t} a^{(1)}(s) d s \sigma_{3}\right\}$. The corresponding transformed Hamiltonian is given by

$$
H_{1}(t)=\left(\begin{array}{cc}
0 & b_{1}(t) \\
c_{1}(t) & 0
\end{array}\right)
$$

where

$$
b_{1}(t):=b^{(1)}(t) e^{i \gamma_{1}(t)}, \quad c_{1}(t):=c^{(1)}(t) e^{-i \gamma_{1}(t)}, \quad \gamma_{1}(t):=2 \int_{0}^{t} a^{(1)}(s) d s
$$

The evolution operator $U_{1}$ of $H_{1}$ is related to the evolution operator of the original Hamiltonian $H$ according to

$$
U_{1}(t)=\exp \left(i \int_{0}^{t} a^{(1)}(s) d s \sigma_{3}\right) U^{(0)}(t)^{\dagger} U(t)
$$

where we have used Eq. (22). 
Now since $H_{1}$ has the same form as $H$, we can repeat the above analysis using $H_{1}$ in place of $H$. Performing an adiabatic canonical transformation on $H_{1}$ we obtain the transformed Hamiltonian

$$
H_{1}^{(1)}(t)=\left(\begin{array}{cc}
a_{1}^{(1)}(t) & b_{1}^{(1)}(t) \\
c_{1}^{(1)} & -a_{1}^{(1)}(t)
\end{array}\right),
$$

where

$$
\begin{gathered}
a_{1}^{(1)}(t):=E_{1}^{(1)}(t) \cos \eta_{1}(t), \quad b_{1}^{(1)}(t):=f_{1,0}^{-1} E_{1}^{(1)} \sin \eta_{1}(t), \\
c_{1}^{(1)}(t):=f_{1,0} E_{1}^{(1)}(t) \sin \eta_{1}(t), \quad E_{1}^{(1)}(t):=E^{(1)}(t) \cos \eta(t), \\
f_{1}(t):=i \sqrt{\frac{c_{1}(t)}{b_{1}(t)}}=i f_{0} e^{-i \gamma_{1}(t)}, \quad f_{1,0}:=f_{1}(0)=i f_{0}, \\
\eta_{1}(t):=2 \int_{0}^{t} E^{(1)}(s) \sin \eta(s) d s .
\end{gathered}
$$

Clearly we can repeat this procedure indefinitely and construct an infinite product expansion for the evolution operator. Again if we compute only a finite number of terms in this expansion, then we obtain a generalization of the adiabatic approximation. The validity of this approximation may be checked by computing the transformed Hamiltonians. It is not difficult to show that the transformed Hamiltonian obtained after $\ell$ adiabatic canonical transformations is of the form

$$
H_{\ell}^{(1)}(t)=h_{\ell}(t) S(t),
$$

where

$$
\begin{gathered}
H_{\ell}(t)=E^{(1)}(t) \cos \eta(t) \cos \eta_{1}(t) \cos \eta_{2}(t) \cdots \cos \eta_{\ell-1}(t), \\
\eta_{j}(t):=2 \int_{0}^{t} E^{(1)}(s) \cos \eta(s) \cos \eta_{1}(s) \cdots \cos \eta_{j-1}(s) \sin \eta_{j-1}(s) d s,
\end{gathered}
$$

where $j \in\{2,3, \ldots, \ell-1\}$ and $S(t)$ is a two-by-two matrix of unit determinant. Clearly if for some $\ell, h,(t)$ is negligible, then the above-mentioned generalization of the adiabatic approximation is valid.

Finally let us note that in general the initial Hamiltonian (29) can be written in the form

$$
H(t)=\alpha_{1}(t) \sigma_{1}+\alpha_{2}(t) \sigma_{2}+a(t) \sigma_{3},
$$

with $\alpha_{1}=(b+c) / 2$ and $\alpha_{2}=i(b-c) / 2$. Performing the canonical transformation (21) defined by $g(t)=\exp \left\{i \int_{0}^{t} \alpha_{2}(s) d s \sigma_{2}\right\}$, we transform the Hamiltonian (54) into

$$
H^{\prime}(t)=\alpha^{\prime}(t) \sigma_{1}+a^{\prime}(t) \sigma_{3}=\left(\begin{array}{cc}
a^{\prime}(t) & \alpha^{\prime}(t) \\
\alpha^{\prime}(t) & -a^{\prime}(t)
\end{array}\right),
$$

where

$$
\begin{gathered}
\alpha^{\prime}(t):=\alpha_{1}(t) \cos \xi(t)-a(t) \sin \xi(t), \quad a^{\prime}(t):=\alpha_{1}(t) \sin \xi(t)+a(t) \cos \xi(t), \\
\xi(t):=\int_{0}^{t} \alpha_{2}(s) d s .
\end{gathered}
$$


Here we have used Eqs. (21) and (46). Next we perform another canonical transformation, namely the one defined by $g(t)=\exp \left\{i \int_{0}^{t} a^{\prime}(s) d s \sigma_{3}\right\}$. This transformation maps the Hamiltonian (55) into

$$
\begin{aligned}
H^{\prime \prime}(t) & =\alpha^{\prime}(t) e^{i \eta^{\prime}(t) \sigma_{3} / 2} \sigma_{1} e^{-i \eta^{\prime}(t) \sigma_{3} / 2} \\
& =\alpha^{\prime}(t)\left[\cos \eta^{\prime}(t) \sigma_{1}-\sin \eta^{\prime}(t) \sigma_{2}\right]=\alpha^{\prime}(t)\left(\begin{array}{cc}
0 & e^{i \eta^{\prime}(t)} \\
e^{-i \eta^{\prime}(t)} & 0
\end{array}\right),
\end{aligned}
$$

where $\eta^{\prime}(t):=2 \int_{0}^{t} a^{\prime}(s) d s$. This Hamiltonian is not only a member of Class 3 Hamiltonians, but initially (at $t=0$ ) its off-diagonal matrix elements are equal. In particular, it has the properties 1 in the above list. Note that we can carry out these canonical transformations on any two-level Hamiltonian. Therefore, every two-level Hamiltonian is canonically equivalent to a Class 3 Hamiltonian of the form (56). This means that the results obtained for Class 3 Hamiltonians apply to arbitrary two-level Hamiltonians.

\section{TIME-DEPENDENT SIMPLE HARMONIC OSCILLATOR}

It is well-known that the solution of every second-order linear $\mathrm{ODE}^{23}$ can be reduced to the classical equation of motion for a simple harmonic oscillator with a time-dependent frequency $\omega=\omega(t)$,

$$
\ddot{x}(t)+\omega^{2}(t) x(t)=0 .
$$

It is also well-known that one can reduce both the classical and quantum equations of motion for a generalized harmonic oscillator to Eq. (57). ${ }^{24,25,22,26}$ This equation has, therefore, many physical applications. ${ }^{25,27}$ Yet an exact analytic expression for the general solution of this equation is not known even for the case of real frequency. ${ }^{28}$ The lack of an exact analytic solution of Eq. (57) is not surprising. One way to see this is to recall that the time-independent Schrödinger equation for an arbitrary potential $V(x)$ in one dimension is given by

$$
\frac{d^{2} \psi_{n}}{d x^{2}}+\left(\frac{\hbar^{2}\left[E_{n}-V(x)\right]}{2 m}\right) \psi_{n}=0,
$$

where $E_{n}$ and $\psi_{n}$ are the energy eigenvalues and eigenfunctions, respectively. Equation (58) can be easily identified with Eq. (57) provided that one makes the change of variables: $x \rightarrow t, \psi_{n}$ $\rightarrow x$, and $\left\{\hbar^{2}[E-V(x)]\right\} /(2 m) \rightarrow \omega^{2}(t)$. This shows that if one was able to find the exact analytic solution of Eq. (57) for arbitrary frequency $\omega$, then one would have been able to find the general solution of the time-independent Schrödinger equation for any potential $V$.

In the following we shall consider the case of an ordinary time-dependent harmonic oscillator (57) with real frequency. In order to apply the results of Sec. IV to Eq. (57), we first express it in the form of a system of first-order ODEs. Defining,

$$
|\psi(t)\rangle:=\left(\begin{array}{c}
x(t) \\
v(t)
\end{array}\right), \quad v(t):=\dot{x}(t)
$$

we can write Eq. (57) in the form of the Schrödinger equation (1) with a two-level Hamiltonian of the form (29) with

$$
a=0, \quad b=i, \quad c=-i \omega(t)^{2}, \quad E=\omega(t) .
$$


Since $a=0$, this system belongs to the Class 3 of Sec. IV with

$$
\begin{gathered}
f(t)=\omega(t), \quad \eta(t)=2 \int_{0}^{t} \omega(s) d s \\
H^{(1)}=E^{(1)}(t)\left(\begin{array}{cc}
\cos \eta(t) & \frac{\sin \eta(t)}{\omega_{0}} \\
\omega_{0} \sin \eta(t) & \cos \eta(t)
\end{array}\right), \quad E^{(1)}(t):=\frac{i \dot{\omega}(t)}{2 \omega(t)} .
\end{gathered}
$$

Clearly, for real frequency $\omega(t)$ we can scale the time variable $t$ so that $\omega_{0}=1$. Then the transformed Hamiltonian (60) takes the form

$$
H^{(1)}(t)=E^{(1)}(t)\left[\sin \eta(t) \sigma_{1}+\cos \eta(t) \sigma_{3}\right]=E^{(1)}(t) e^{-i \eta(t) \sigma_{2} / 2} \sigma_{3} e^{i \eta(t) \sigma_{2} / 2} .
$$

Note that since $\eta(t)$ is real, the Hamiltonian (61) is an anti-Hermitian matrix with orthogonal eigenvectors. Therefore, up to a factor of $i$ it describes a two-level spin system with a SU(2) dynamical group. ${ }^{19,3}$ [Note that one can absorb the factor of $i$ in the definition of the time variable $t$, i.e., by defining the imaginary time variable $\tau:=-i t$. Therefore, the dynamics given by the Hamiltonian (61) may be viewed as the dynamics of a spin system with imaginary time.] This is rather surprising, for it is well-known that the quantum harmonic oscillator has $\mathrm{SU}(1,1)$ dynamical group and that its Schrödinger equation may be reduced to Eq. (57) by means of a quantum canonical transformation corresponding to a time-dependent dilatation. ${ }^{26}$

In view of the fact that $E^{(1)}$ is proportional to the derivative of $\ln \omega$, we can make a change of independent variable, namely $t \rightarrow \eta$. Note that $\eta$ is the integral of a positive real function of $t$. Hence, it is a monotonically increasing function of $t$. Making this change of variable the Schrödinger equation for the Hamiltonian (61) becomes

$$
i \frac{d}{d \eta} \widetilde{U}(\eta)=\widetilde{H}(\eta) \widetilde{U}(\eta), \quad \widetilde{U}(\eta)=1
$$

where $\widetilde{U}(\eta):=U^{\prime}(t(\eta)), U^{\prime}(t)$ is the evolution operator for the the Hamiltonian (61),

$$
\begin{gathered}
\widetilde{H}(\eta):=\widetilde{E}(\eta) e^{-i \eta \sigma_{2} / 2} \sigma_{3} e^{i \eta \sigma_{2} / 2}=\widetilde{E}(\eta)\left(\sin \eta \sigma_{1}+\cos \eta \sigma_{3}\right), \\
\widetilde{E}(\eta):=\frac{i \omega^{\prime}(\eta)}{2 \omega(\eta)}, \quad \omega^{\prime}:=\frac{d \omega}{d \eta} .
\end{gathered}
$$

Up to a factor of $i$, the Hamiltonian (62) describes the interaction of a spin 1/2 magnetic dipole with a changing magnetic field whose direction rotates uniformly in the $x-z$ plane.

As we mentioned in Sec. IV for the Class 3 systems $H^{(2)}(t)=H(t)$. Hence direct application of the method of the adiabatic product expansion does not lead to a solution of the Schrödinger equation for the Hamiltonian (61) or (62). In this case, either one constructs the modified adiabatic product expansion of Sec. IV or examines the adiabatic series expansion of Ref. 2. The latter yields a series expansion for the evolution operator $\widetilde{U}(\eta)$ of the Hamiltonian $\widetilde{H}(\eta)$, namely

$$
\begin{aligned}
\widetilde{U}(\eta)= & \mathcal{T} \exp \left(-i \int_{0}^{\eta} \widetilde{H}(s) d s\right) \\
= & \left.1-i \int_{0}^{\eta} \widetilde{H}(s) d s+\frac{(-i)^{2}}{2} \int_{0}^{\eta} \int_{0}^{\eta} \mathcal{T} \widetilde{H}\left(s_{1}\right) \widetilde{H}\left(s_{2}\right)\right] d s_{1} d s_{2}+\cdots+\frac{(-i)^{n}}{n !} \\
& \left.\times \int_{0}^{\eta} \cdots \int_{0}^{\eta} \mathcal{T} \widetilde{H}\left(s_{1}\right) \cdots \widetilde{H}\left(s_{n}\right)\right] d s_{1} \cdots d s_{n}+\cdots,
\end{aligned}
$$


where $\mathcal{T}$ stands for the time-ordering operator. Since $\widetilde{H}(\eta)$ is proportional to $\omega^{\prime}(\eta)$, for slowly varying $\omega$ one obtains an approximate expression for $\widetilde{U}(\eta)$ by computing a finite number of terms in this series. This is in fact another generalization of the adiabatic approximation, because if one keeps only the first term in this series and neglects the other terms one is essentially neglecting $\widetilde{H}$ or alternatively $H^{(1)}$. As we explained above, this is just the adiabatic approximation. If one keeps more terms in this series, then one obtains a better approximation than the adiabatic approximation.

\section{QUADRUPOLE INTERACTION OF A SPIN 1 PARTICLE WITH A CHANGING ELECTRIC FIELD}

Consider a spin 1 particle interacting with a changing electric field $\overrightarrow{\mathcal{E}}(t)$ $=\left(\mathcal{E}_{1}(t), \mathcal{E}_{2}(t), \mathcal{E}_{3}(t)\right)$ according to the Stark Hamiltonian

$$
H(t)=\lambda[\vec{J} \cdot \overrightarrow{\mathcal{E}}(t)]^{2},
$$

where $\lambda$ is a real coupling constant and $\vec{J}$ is the angular momentum of the particle. The quadrupole interactions of the form (65) have been extensively studied for fermionic systems in relation with the non-Abelian geometric phases ${ }^{29-31}$ (See also Ref. 32.) The occurrence of non-Abelian geometric phases for the degenerate spin 1 systems has been pointed out in Ref. 33. For these systems, the particle has a definite angular momentum $j=1$ and the Hamiltonian is a $3 \times 3$ matrix. Using the spin $j=1$ representation of $J_{i}$, we can express the Stark Hamiltonin (65) in the form

$$
H=\left(\frac{\lambda r^{2}}{2}\right)\left(\begin{array}{ccc}
1+2 z^{2} & \sqrt{2} z e^{-i \theta} & e^{-2 i \theta} \\
\sqrt{2} z e^{i \theta} & 2 & -\sqrt{2} z e^{-i \theta} \\
e^{2 i \theta} & -\sqrt{2} z e^{i \theta} & 1+2 z^{2}
\end{array}\right),
$$

where $r, \theta$, and $z$ are defined by

$$
r:=\sqrt{\mathcal{E}_{1}^{2}+\mathcal{E}_{2}^{2}}, \quad e^{i \theta}:=\frac{\mathcal{E}_{1}+i \mathcal{E}_{2}}{r}, \quad z:=\frac{\mathcal{E}_{3}}{r} .
$$

In view of the general results of Ref. 33, if $r \neq 0$ then the Hamiltonian (66) has a degenerate and a nondegenerate eigenvalue. In the following we shall consider the case where $\mathcal{E}_{3}=0$. The general case $\mathcal{E}_{3} \neq 0$ can be similarly treated.

If $\mathcal{E}_{3}=0$, then $z=0$ and

$$
H=\left(\frac{\lambda r^{2}}{2}\right)\left(\begin{array}{ccc}
1 & 0 & e^{-2 i \theta} \\
0 & 2 & 0 \\
e^{2 i \theta} & 0 & 1
\end{array}\right)
$$

The eigenvalues of this Hamiltonian are given by

$$
E_{1}=0, \quad E_{2}=\lambda r^{2} .
$$

For $r \neq 0, E_{1}$ is nondegenerate and $E_{2}$ is doubly degenerate. A set of orthonormal eigenvectors of this Hamiltonian is given by

$$
\left|\psi_{1} ; R\right\rangle:=\frac{1}{\sqrt{2}}\left(\begin{array}{c}
-1 \\
0 \\
e^{2 i \theta}
\end{array}\right), \quad\left|\psi_{2}, 1 ; R\right\rangle:=\frac{1}{\sqrt{2}}\left(\begin{array}{c}
1 \\
0 \\
e^{2 i \theta}
\end{array}\right), \quad\left|\psi_{2}, 2 ; R\right\rangle:=\left(\begin{array}{l}
0 \\
1 \\
0
\end{array}\right),
$$

where $R=(r, \theta)$. 
Next we compute $U^{(0)}(t)$ for this system. In order to do this we first use Eq. (15) to calculate $\mathcal{A}^{n}$. In view of the fact that the Hamiltonian (67) is Hermitian, $\left|\phi_{n}, a ; R\right\rangle=\left|\psi_{n}, a ; R\right\rangle$ and Eq. (15) leads to

$$
\mathcal{A}^{1}=-d \theta, \quad \mathcal{A}^{2}=\left(\begin{array}{cc}
-d \theta & 0 \\
0 & 0
\end{array}\right)
$$

Substituting Eqs. (70) into Eq. (14) and making use of Eq. (68), we find

$$
K^{1}(t)=e^{-i\left[\theta(t)-\theta_{0}\right]}, \quad K^{2}(t)=e^{-i \rho(t)}\left(\begin{array}{cc}
e^{-i\left[\theta(t)-\theta_{0}\right]} & 0 \\
0 & 1
\end{array}\right),
$$

where

$$
\theta_{0}:=\theta(0), \quad \rho(t):=\lambda \int_{0}^{t} r(s)^{2} d s
$$

Using Eqs. (18) and (69), we have

$$
U^{(0)}(t)=\frac{1}{2}\left(\begin{array}{ccc}
\left(1+e^{-i \rho(t)}\right) e^{i \theta_{-}(t)} & 0 & \left(-1+e^{-i \rho(t)}\right) e^{-i \theta_{+}(t)} \\
0 & 2 e^{-i \rho(t)} & 0 \\
\left(-1+e^{-i \rho(t)}\right) e^{i \theta_{+}(t)} & 0 & \left(1+e^{-i \rho(t)}\right) e^{i \theta_{-}(t)}
\end{array}\right),
$$

where $\theta_{ \pm}(t):=\theta(t) \pm \theta_{0}$.

Next we compute the Hamiltonian $H^{(1)}(t)$. This involves the calculation of $A_{a b}^{n m}(t)$ and $H_{a b}^{m n}(t)$ for $m \neq n$. Using Eqs. (24) and (69) we have

$$
\begin{gathered}
A_{11}^{12}=A_{11}^{21}=-\dot{\theta}, \quad A_{12}^{12}=A_{21}^{21}=0, \\
H_{11}^{21}=H_{11}^{12}=\dot{\theta} e^{i \rho(t)}, \quad H_{12}^{12}=H_{21}^{21}=0 .
\end{gathered}
$$

Substituting these equations in Eq. (23) and using Eq. (69), we obtain

$$
H^{(1)}(t)=-\dot{\theta}(t)\left(\begin{array}{ccc}
\cos \rho(t) & 0 & -i \sin \rho(t) \\
0 & 0 & 0 \\
i \sin \rho(t) & 0 & -\cos \rho(t)
\end{array}\right)=-\dot{\theta}(t)\left[\sin \rho(t) \Sigma_{2}+\cos \rho(t) \Sigma_{3}\right]
$$

where

$$
\Sigma_{2}:=\left(\begin{array}{ccc}
0 & 0 & -i \\
0 & 0 & 0 \\
i & 0 & 0
\end{array}\right), \quad \Sigma_{3}:=\left(\begin{array}{ccc}
1 & 0 & 0 \\
0 & 0 & 0 \\
0 & 0 & -1
\end{array}\right)
$$

It is not difficult to recognize $\Sigma_{2}$ and $\Sigma_{3}$ as the Pauli matrices $\sigma_{2}$ and $\sigma_{3}$ represented in a ( 0 $+1 / 2)$ representation of $\mathrm{SU}(2)$. In view of this identification we can express $H^{(1)}(t)$ in the form

$$
H^{(1)}(t)=-\dot{\theta}(t) e^{i \rho(t) \Sigma_{1} / 2} \Sigma_{3} e^{-i \rho(t) \Sigma_{1} / 2},
$$

where 


$$
\Sigma_{1}:=\left(\begin{array}{lll}
0 & 0 & 1 \\
0 & 0 & 0 \\
1 & 0 & 0
\end{array}\right),
$$

and we have used Eq. (46).

The Hamiltonian $H^{(1)}(t)$ has the following interesting properties.

(a) For $\theta=0$, i.e., $\theta=$ constant, the adiabatic approximation is exact and $U(t)=U^{(0)}(t)$.

(b) In view of Eq. (76) for $\dot{\theta} \neq 0, H^{(1)}(t)$ has three nondegenerate eigenvalues, namely $-\dot{\theta}, 0$, and $\dot{\theta}$. This is quite remarkable because it shows that the adiabatic canonical transformation maps the degenerate Hamiltonian (67) into the nondegenerate Hamiltonian (76).

(c) Since $\Sigma_{i}$ is a representation of the Pauli matrix $\sigma_{i}$, the Hamiltonian (76) belongs to a representation of the Lie algebra of SU(2). This means that one can reduce the Schrödinger equation for this Hamiltonian to that of the dipole Hamiltonian ${ }^{2,3}$

$$
H_{\mathrm{dp}}=-2 \dot{\theta}(t) e^{i \rho(t) J_{1}} J_{3} e^{-i \rho(t) J_{1}} .
$$

(d) We can perform another canonical transformation, namely the one defined by $g(t)$ $=\exp \left[-i \rho(t) \Sigma_{1} / 2\right]$ to transform the Hamiltonian (76) into

$$
H^{(1)^{\prime}}(t)=\frac{r(t)^{2}}{2} \Sigma_{1}-\dot{\theta}(t) \Sigma_{3},
$$

where we have used Eqs. (76), (21), and (72). In particular if $\dot{\theta}$ and $r^{2}$ happen to be proportional, i.e., for some $c \in \mathbb{R}$

$$
\dot{\theta}(t)=\operatorname{cr}(t)^{2},
$$

then $H^{(1)^{\prime}}(t)=r(t)^{2}\left(\frac{1}{2} \Sigma_{1}-c \Sigma_{3}\right)$. In this case the eigenvectors of $H^{(1)^{\prime}}(t)$ are constant and the adiabatic approximation yields the exact solution of the Schrödinger equation for $H^{(1)^{\prime}}(t)$. The corresponding evolution operator is then given by

$$
U^{(1)^{\prime}}(t)=\exp \left(i\left(\frac{1}{2} \Sigma_{1}-c \Sigma_{3}\right) \int_{0}^{t} r(s)^{2} d s\right) .
$$

Having obtained the evolution operator for $H^{(1)^{\prime}}(t)$ we can use Eq. (22) to obtain the evolution operator for $H^{(1)}(t)$ and $H(t)$. This yields the following expression for the evolution operator for $H(t)$ :

$$
U(t)=U^{(0)}(t) e^{i \rho(t) \Sigma_{1} / 2} U^{(1)^{\prime}}(t),
$$

where $U^{(0)}(t)$ and $U^{(1)^{\prime}}(t)$ are given by Eqs. (73) and (79).

The above analysis shows that the condition (78) defines a class of exactly solvable timedependent Stark Hamiltonians. If $\theta=\omega t$, for some constant frequency $\omega$, this condition corresponds to the case of the rotating electric field $\vec{E}=r(\sin \omega t, \cos \omega t, 0)$ with magnitude $r$.

\section{CONCLUSION}

In this article we have extended the method of the adiabatic product expansion to nonHermitian and degenerate Hamiltonians. We showed that in general there were three possibilities for the adiabatic product expansion.

(1) The expansion terminates after a finite number of iteractions. This happens when one of the transformed Hamiltonians vanishes. In this case the method yields the exact solution for the Schrödinger equation. 
(2) The expansion consists of an infinite number of distinct terms. In this case, the method does not lead to an exact solution, but it gives rise to a generalization of the adiabatic approximation. This approximation is performed by keeping a finite number of terms in the product expansion. The general asymptotic behavior of the adiabatic product expansion has not been studied. However, one can interpret this approximation by recalling that the condition for the termination of the product expansion corresponds to the validity of the conventional adiabatic approximation for one of the transformed Hamiltonians.

(3) The expansion involves terms which are not distinct. In this case the expansion does not lead to a solution. However, usually one can make another time-dependent canonical transformation after each adiabatic transformation and obtain an infinite product expansion with the properties of case (2) above.

We have considered some specific problems that one can attempt to solve using this method. We treated the case of a general nondegenerate two-level system and applied our general results to the more specific case of the classical equation of motion for a harmonic oscillator with a timedependent frequency. In this case, we showed that the adiabatic canonical transformation mapped the corresponding two-level quantum system to a quantum system with an anti-Hermitian Hamiltonian. Although the direct application of the method of adiabatic product expansion did not yield a solution, we could construct the modified adiabatic product expansion. We have also outlined an adiabatic series expansion for the time-evolution operator of this system which led to another generalization of the adiabatic approximation. Finally, we considered the application of our method to treat the quadrupole interaction of a spin 1 particle with a changing electric field. The corresponding (Stark) Hamiltonian had a nondegenerate as well as a degenerate eigenvalue. We showed that the adiabatic canonical transformation mapped this Hamiltonian to a Hamiltonian which had nondegenerate eigenvalues and belonged to a reducible $(0+1 / 2)$ representation of the Lie algebra of SU(2). This means that we can directly use the results of Refs. 2 and 3 which treat the Schrödinger equation for a nondegenerate Hamiltonian belonging to (an irreducible representation of) the Lie algebra of SU(2). Furthermore, we identified a class of exactly solvable spin 1 quadruple Hamiltonians.

\footnotetext{
${ }^{1}$ A. Mostafazadeh, Phys. Lett. A 228, 7 (1997).

${ }^{2}$ A. Mostafazadeh, Phys. Rev. A 55, 1653 (1997).

${ }^{3}$ A. Mostafazadeh, J. Math. Phys. 38, 3489 (1997)

${ }^{4}$ A. Mostafazadeh, Phys. Rev. A 55, 4084 (1997).

${ }^{5}$ H. C. Baker, Phys. Rev. Lett. 50, 1579 (1983); A. Siegma, Opt. Commun. 31, 369 (1979); G. Dattoli, T. Hermsen, L. Mezi, and A. Torrr, Phys. Rev. A 37, 4334 (1988).

${ }^{6}$ G. Dattoli, A. Torrr, and R. Mignani, Phys. Rev. A 42, 1467 (1990).

${ }^{7}$ A. Kvitsinsky and S. Putterman, J. Math. Phys. 32, 1403 (1991).

${ }^{8}$ J. Wong, J. Math. Phys. 8, 2039 (1967).

${ }^{9}$ J. C. Garrison and E. M. Wright, Phys. Lett. A 128, 177 (1988).

${ }^{10}$ M. V. Berry, Proc. R. Soc. London, Ser. A 392, 45 (1984).

${ }^{11}$ G. Dattoli, R. Mignani, and A. Torre, J. Phys. A 23, 5795 (1990).

${ }^{12}$ Ch. Miniature, C. Sire, J. Baudon, and J. Bellissard, Europhys. Lett. 13, 199 (1990).

${ }^{13}$ A. Mondragón and E. Hernández, J. Phys. A 29, 2567 (1996).

${ }^{14}$ A. Mostafazdeh, Koç University, preprint.

${ }^{15}$ M. Born and V. Fock, Z. Phys. 51, 165 (1928).

${ }^{16}$ T. Kato, J. Phys. Soc. Jpn. 5, 435 (1950).

${ }^{17}$ G. Nenciu and G. Rasche, J. Phys. A 25, 5741 (1992).

${ }^{18}$ F. Wilczek and A. Zee, Phys. Rev. Lett. 52, 2111 (1984).

${ }^{19}$ S. J. Wang and W. Zuo, Phys. Lett. A 196, 13 (1994).

${ }^{20}$ S. V. Prants, J. Phys. A 19, 3657 (1986); Y.-Z. Lai, J.-Q. Liang, H. J. W. Müller-Kirsten, and J.-G. Zhuo, ibid. 29, 1773 (1996); S. Zhang and F. Li, ibid. 29, 6143 (1996).

${ }^{21}$ S. J. Wang, W. Zuo, A. Weiguny, and F. L. Li, Phys. Lett. A 196, 7 (1994); V. Penna, Ann. Phys. (N.Y.) 245, 389 (1996).

${ }^{22}$ R. Jackiw, Int. J. Mod. Phys. A 3, 285 (1988).

${ }^{23}$ E. L. Ince, Ordinary Differential Equations (Dover, New York, 1956).

${ }^{24}$ K. Husimi, Prog. Theor. Phys. 9, 381 (1953).

${ }^{25}$ V. V. Dodonov and M. A. Mańko, in Invariants and the Evolution of Nonstationary Quantum Systems, edited by M. A. Markov (Nova, Commack, NY, 1989).

${ }^{26}$ A. Mostafazadeh, J. Phys. A 31, 6495 (1998).
} 
${ }^{27}$ W. Paul, Rev. Mod. Phys. 62, 531 (1990); F. Diedrich, J. C. Bergquist, W. M. Itano, and D. J. Wineland, Phys. Rev. Lett. 62, 403 (1989); D. J. Heinzen and D. J. Wineland, Phys. Rev. A 42, 2977 (1990); G. S. Agrawal and S. Arun Kumar, Phys. Rev. Lett. 67, 3665 (1991).

${ }^{28}$ R. Bellman, Perturbation Techniques in Mathematics, Physics, and Engineering (Holt, Rinehart and Winston, New York, 1964).

${ }^{29}$ C. A. Mead, Phys. Rev. Lett. 59, 161 (1987).

${ }^{30}$ J. E. Avron, L. Sadun, J. Segret, and B. Simon, Phys. Rev. Lett. 61, 1329 (1988).

${ }^{31}$ J. E. Avron, L. Sadun, J. Segret, and B. Simon, Commun. Math. Phys. 124, 595 (1989).

${ }^{32}$ M. T. Johnsson and I. J. Aitchison, J. Phys. A 30, 2085 (1997).

${ }^{33}$ A. Mostafazadeh, J. Phys. A 30, 7525 (1997). 